\title{
Libros de matemáticas con estrellas Michelin
}

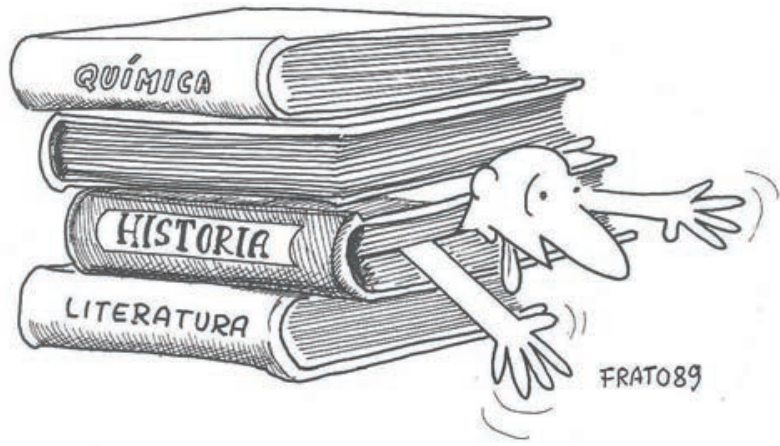

NO TE PREOCUPES.

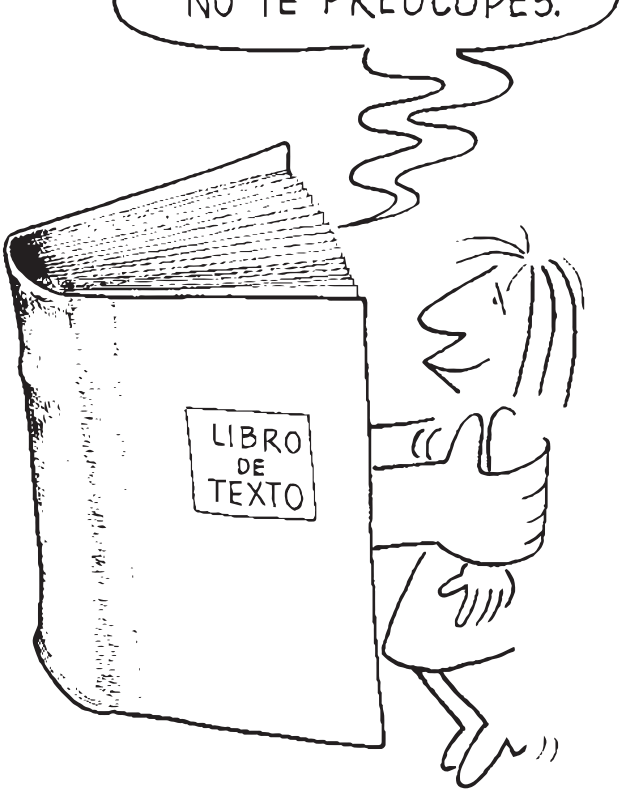

Este artículo aborda el controvertido tema del uso del libro de texto de matemáticas en el aula. Controvertido porque, aunque es un recurso didáctico más al servicio de la enseñanza, a veces bloquea la entrada de otras metodologías.

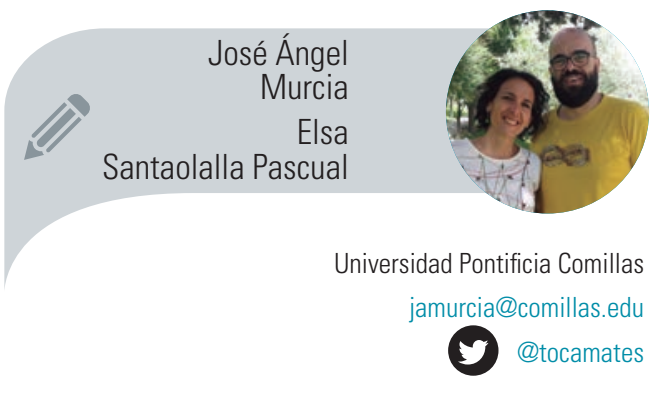
Se dan una serie de criterios sobre lo que los autores consideran que debería ser un buen libro de texto. No descartamos que estos consejos pudieran servir también a las editoriales que, aunque muestran muchas ganas de ofrecer productos interesantes y novedosos, no siempre lo consiguen. 
"¿Qué libro de texto recomiendas?" "¿qué hago si en mi centro me 'imponen' usar uno?". Estas son algunas de las preguntas que nos hacen tanto en los cursos de formación continua como en las clases de formación inicial. Y es que queramos o no, los libros de texto de matemáticas son una realidad en las aulas y por eso, los maestros en activo y los estudiantes de magisterio se sienten concernidos por este tema. Algunos porque son conscientes del agobio que supone seguir un libro de texto, más si es "al pie de la letra", otros porque saben que el libro de texto puede servir de excusa para no introducir cambios e innovaciones en sus metodologías.

De todos modos, todos plantean estas preguntas desde el pudor. Parece que esperan que respondamos: "el libro no debería utilizarse". Sin embargo, la verdad es que hace falta dominar la materia que impartes y su didáctica, o ser un poco inconsciente, para plantearse una programación anual que incluya una batería de recursos de todo tipo para afrontar con éxito un curso de enseñanzas obligatorias. Mirémoslo por el otro lado, si no eres un experto en una materia -en la que sealo mejor que puedes hacer es tener un buen manual cerca. Por poner un ejemplo de lo que acabamos de decir, un ingeniero está habilitado para dar clase de matemáticas en secundaria, pero salvo que tenga mucha experiencia, lo mejor que puede pasarle es que tenga cerca un buen libro de texto y sepa cómo usarlo.

La elección de qué libro de texto utilizar no siempre es posible. En muchas ocasiones es el departamento el que toma la decisión, para darle continuidad dentro del centro. Otras veces, el uso de un manual viene decidido por la dirección del centro que tiene un compromiso con esa editorial. En ese caso no hay más remedio que utilizarlo. Además, no es una opción no hacerlo, no se le puede decir a los padres que gasten más de 30 (por materia) para luego ver que no se utiliza ese manual.

Los autores de este artículo hemos tenido la experiencia de colaborar en distintos momentos de nuestras vidas con editoria-

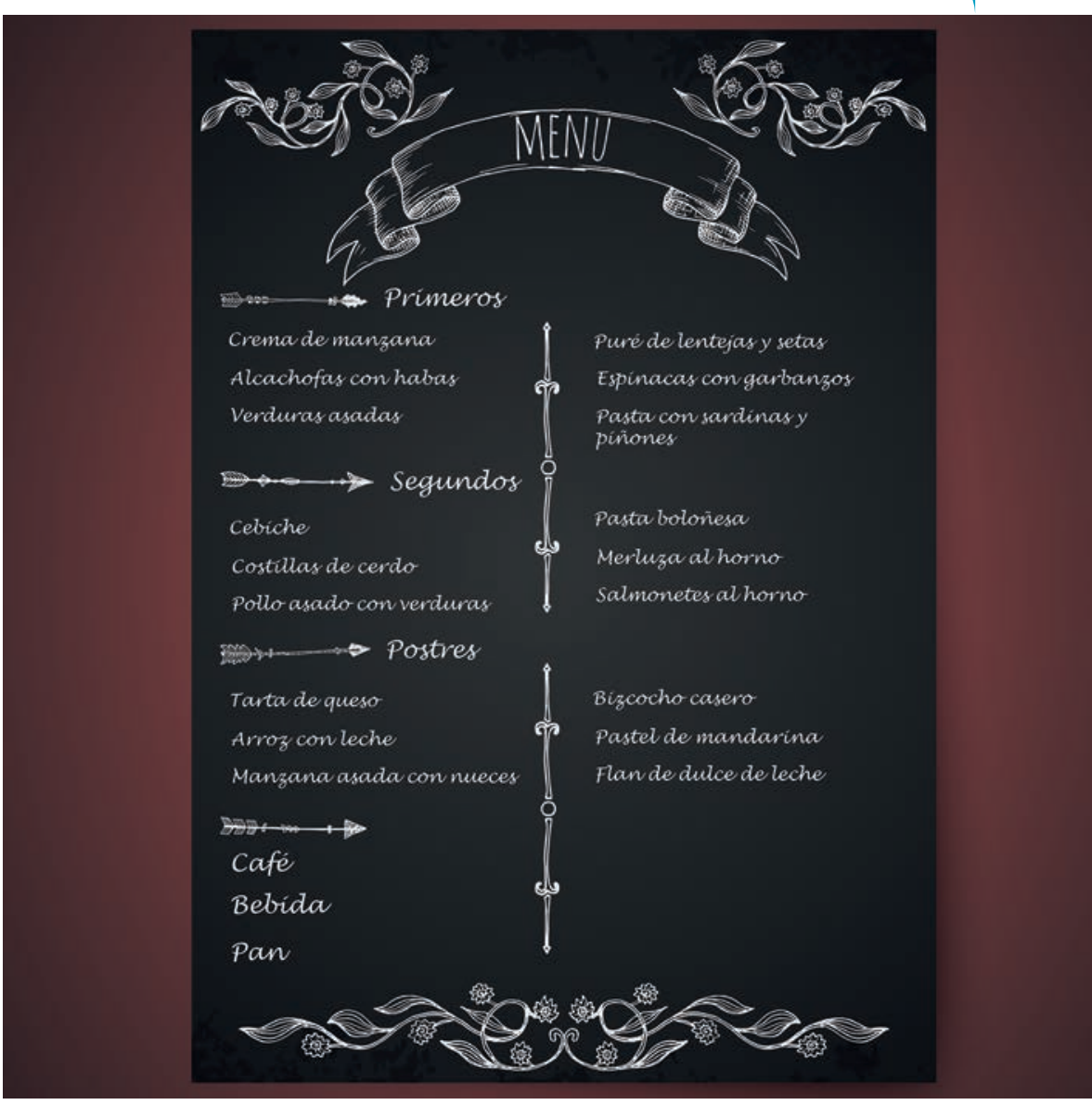

les de libros de texto. A la hora de elaborar, corregir o convertir a digital un proyecto editorial surge una cuestión fundamental ¿cuáles son los contenidos que debe incluir? No es fácil, pues, aunque el Ministerio de Educación fija unas orientaciones a través de diversos reales decretos -para cada etapa educativa-, estos no detallan qué "entra" en cada curso, ni cómo deben impartirse. Bueno, casi, hay una pequeña excepción, las ciudades autónomas de Ceuta y Melilla siguen siendo responsabilidad del Ministerio de Educación (en sus sucesivas acepciones), por lo que las concreciones curriculares que proporciona suelen servir como primera indicación para las editoriales. Pero como luego cada comunidad autónoma organiza sus currículos a su manera -dispar-, las editoriales pueden recurrir a lanzar ediciones adaptadas a esos currículos -algo que acarrea costes- o, en el peor de los casos, igualar el currículo "por arriba", algo que siempre se puede hacer -dar más contenidos que los que vienen en el programa oficial- pero de lo que se debe ser consciente cuando eres el responsable de la asignatura. Este criterio editorial enraizaría con el miedo 


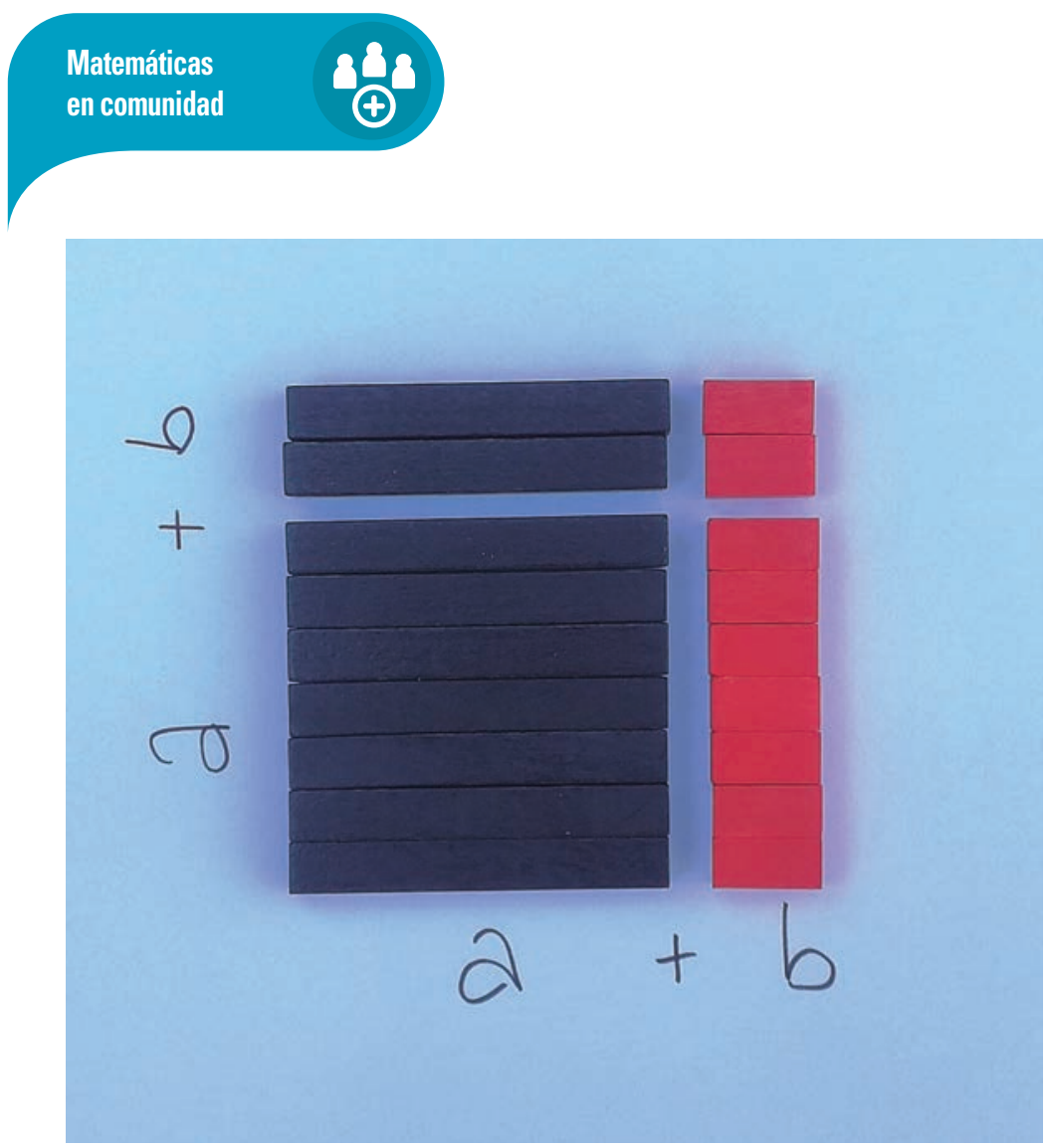

Podemos usar regletas para demostrar que el cuadrado de $(a+b)$ es igual a la suma del cuadrado de a, más el cuadrado de b, más dos veces el producto de $a \times b$ existente a dar la sensación de "bajar el nivel". Ninguna editorial quiere que sus libros sean tachados de "fáciles" y menos en una materia con una tradición de hueso como son las matemáticas. Por poner un ejemplo final en ese sentido, cada editorial mira con lupa los libros de las editoriales que le hacen la competencia, observando, entre otros indicadores, el número de unidades didácticas o el número de ejercicios y problemas que han incluido en su manual ino vaya a ser que el suyo tenga menos!, y estos indicadores, como veremos, no son precisamente los que dan realmente calidad didáctica a un libro de texto de matemáticas.

Pero si se trata de "ser legales", hay algunas ideas que vendría bien tener en cuenta. El currículo de matemáticas es espiral, esto implica que cada año se debe pasar por los mismos contenidos, ampliándolos. Los decretos oficiales así lo imponen, igual que hacen hincapié en que los contenidos de cada bloque deben enlazarse y relacionarse lo que supone que no podemos abordarlos de forma separada y estanca. Además, la propia ley aclara que para facilitar la concreción curricular, los contenidos se han organizado en unos bloques determinados, pero insiste en que "esa" agrupación no debe considerarse "cerrada", sino todo lo contrario e incita a que los docentes (y las editoriales) organicen los contenidos del modo que sea mejor para adecuar la metodología a las necesidades de cada grupo de estudiantes. La cosa parece clara. Hay vía libre para trabajar en el aula como cada uno considere oportuno siempre y cuando, eso sí, se aborden los contenidos de forma enlazada y relacionando unos bloques con otros.

Nos gusta la idea de compararlo con un menú. Supongamos que la aritmética (los números y sus operaciones) fueran los primeros platos; las magnitudes y su medida, los segundos; los contenidos de geometría, los postres; y, finalmente, la probabilidad y la estadística, el café (o la infusión). Aunque hay veces que pedimos dos platos del mismo grupo, lo habitual suele ser tomar un plato de cada grupo. ¡Vaya empacho si comes a base de postres! Gustos aparte, una dieta equilibrada se logra al combinar un plato de cada grupo. En matemáticas pasa lo mismo. Si se dejan las magnitudes y su medida, para trabajarlas todas juntas en sí mismas, por ejemplo, se acaba jempachado de magnitudes! Pero no debemos confundir una dieta equilibrada y diseñada con cabeza, con un "picoteo". No es fácil saber cómo enlazar los contenidos de manera que las conexiones tengan sentido y se aborden desde distintas perspectivas. De hecho, aunque en los primeros cursos de primaria las editoriales tratan de cuidar la "dieta matemática", en cuanto avanzan en la etapa... icalcan el esquema de contenidos que describe la ley!, olvidándose de la parte de la ley que reclama que se aborden de forma enlazada. Basta con observar los índices de los libros de texto de matemáticas para ver que todos siguen el mismo agrupamiento de unidades didácticas por bloques de contenidos como si la estadística o el tratamiento de la información tuviera que darse necesariamente a final de curso, lo que suele ser equivalente a no verlo nunca. ¡Con lo necesario que es saber interpretar las gráficas que aparecen continuamente en las noticias y en la prensa!

Pongamos un ejemplo para que se entienda, la primera noción de fracciones se suele introducir en 3. ${ }^{\circ}$ de primaria, pero 
no se suman hasta que en una vuelta más amplia se tratan en $4 .^{\circ}$, curso en el que solo se suman con igual denominador, en 5. ${ }^{\circ}$ se vuelven a dar y ya se suman fracciones con distinto denominador. A partir de la aparición del concepto en $3 .^{\circ}$ las fracciones vuelven cada año -como el turrónpero cada vez con mayor profundidad. Lo mismo ocurre con el resto de contenidos del curso, van tratándose todos los años con mayor profundidad, además, el orden suele ser siempre el mismo: aritmética (números), más aritmética, aún más aritmética, medida, geometría, estadística y probabilidad. Retomando la analogía del menú, con una programación así diseñada se llega a los postres (la geometría) con pocas ganas, tanto que a veces ni se toma si te has empachado con los platos anteriores, y el café (la probabilidad y estadística), como haya prisa, se deja para otro rato.

La sensación de profesores y alumnos es que todos los años se da lo mismo. La maestra y renovadora de la enseñanza de las matemáticas Maria Antònia Canals participó como autora en unos libros de la editorial Onda -hoy ya descatalogadosen el que todas las unidades didácticas intentaban tratar contenidos de todos los bloques, algo que se hacía además buscando la máxima significancia para los alumnos, con ejemplos cotidianos, juegos, retos y problemas en contextos reales y accesibles. Un menú muy variado. Algo que no siempre ocurre cuando se trata de esta materia.

En los últimos años han surgido algunas propuestas interesantes, a las que nos habría gustado poder dedicar más espacio en estas páginas porque son muy diferentes a las tradicionales. Se trata de EntusiasMAT, ABN, Jump Math y el Método Singapur Si algo tienen en común sus propuestas es que hacen uso intensivo de materiales manipulativos (dados, reglas, palillos...) o imprimibles -en el caso de Jump Math-, materiales que desde hace años todas las editoriales incorporan a sus métodos, pero que no suelen ser invocados desde el libro, convirtiéndose en "juguetes matemáticos" que el maestro

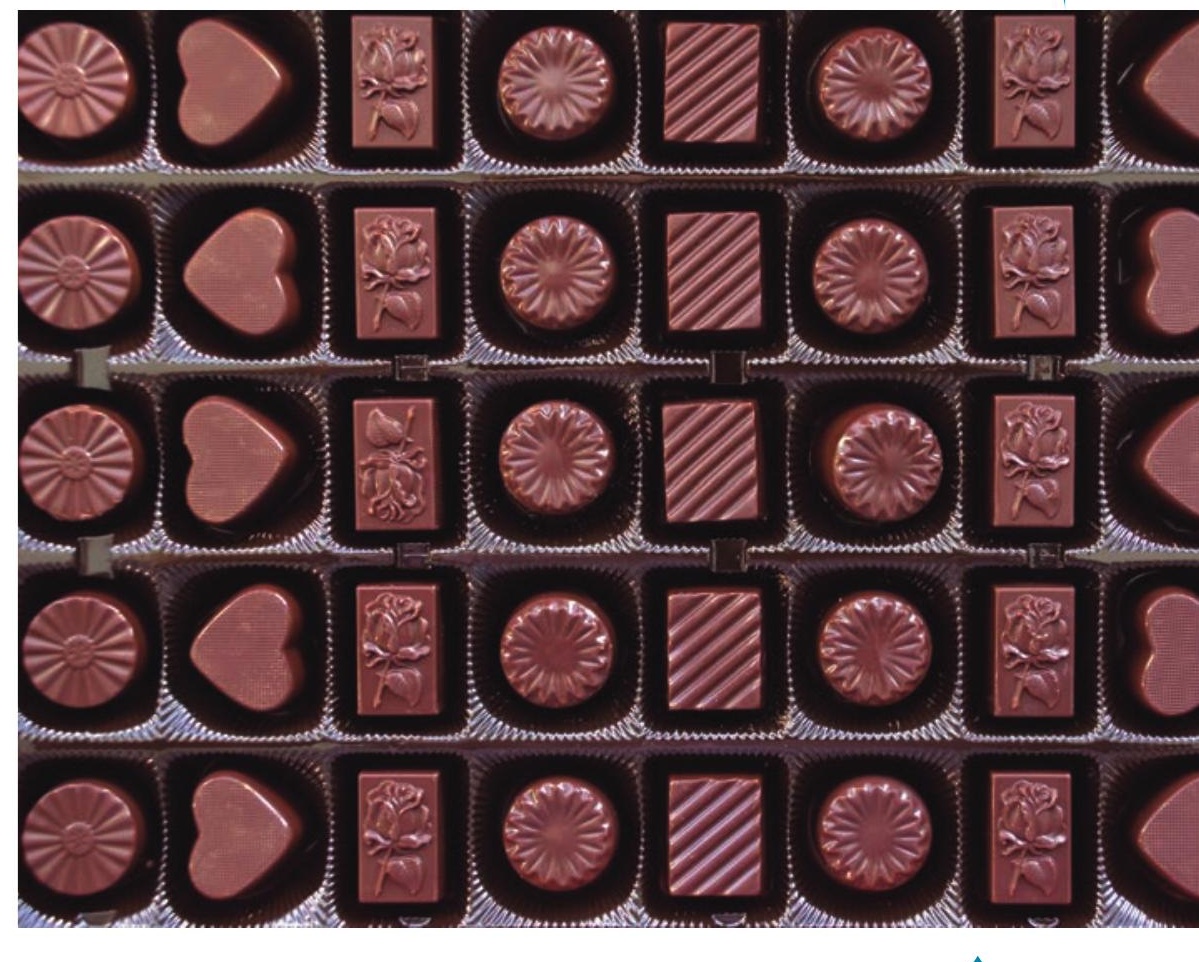

utilizará si sabe cómo, y si no, no los utilizará. En el caso de estas propuestas -muy diferentes entre si- encontramos algo muy positivo: Ilamadas desde el libro a que el alumno lance los dados, construya con los palillos un número (con sus decenas y unidades) o que forme con bloques una forma geométrica... Estas dinámicas obligan a manipular y concretar ideas que suelen contener mucha abstracción para los alumnos.

Desde nuestra modesta experiencia podrían existir ciertos criterios con los que podríamos valorar lo "bueno" que es un libro. Por seguir con nuestra metáfora gastronómica, si pensamos en el libro como en un restaurante, para ver si el libro podría entrar en la lista de candidatos a obtener alguna estrella Michelin, se podría observar si:

* Propone una secuencia espiral y enlazada de los contenidos de los distintos bloques o si no es así, si permite al docente seguir su propio orden (como si estuviera delante de la mesa de un autoservicio/bufé en la que cada uno elige lo que quiere comer según sus preferencias, gustos o necesidades). No podemos contentarnos con un libro que imponga un orden lineal establecido de contenidos inconexos en bloques estancos (como si fueran lentejas, que si quieres las comes y si no...).
Cualquier situación cotidiana nos proporciona un contexto rico para hacer matemáticas 

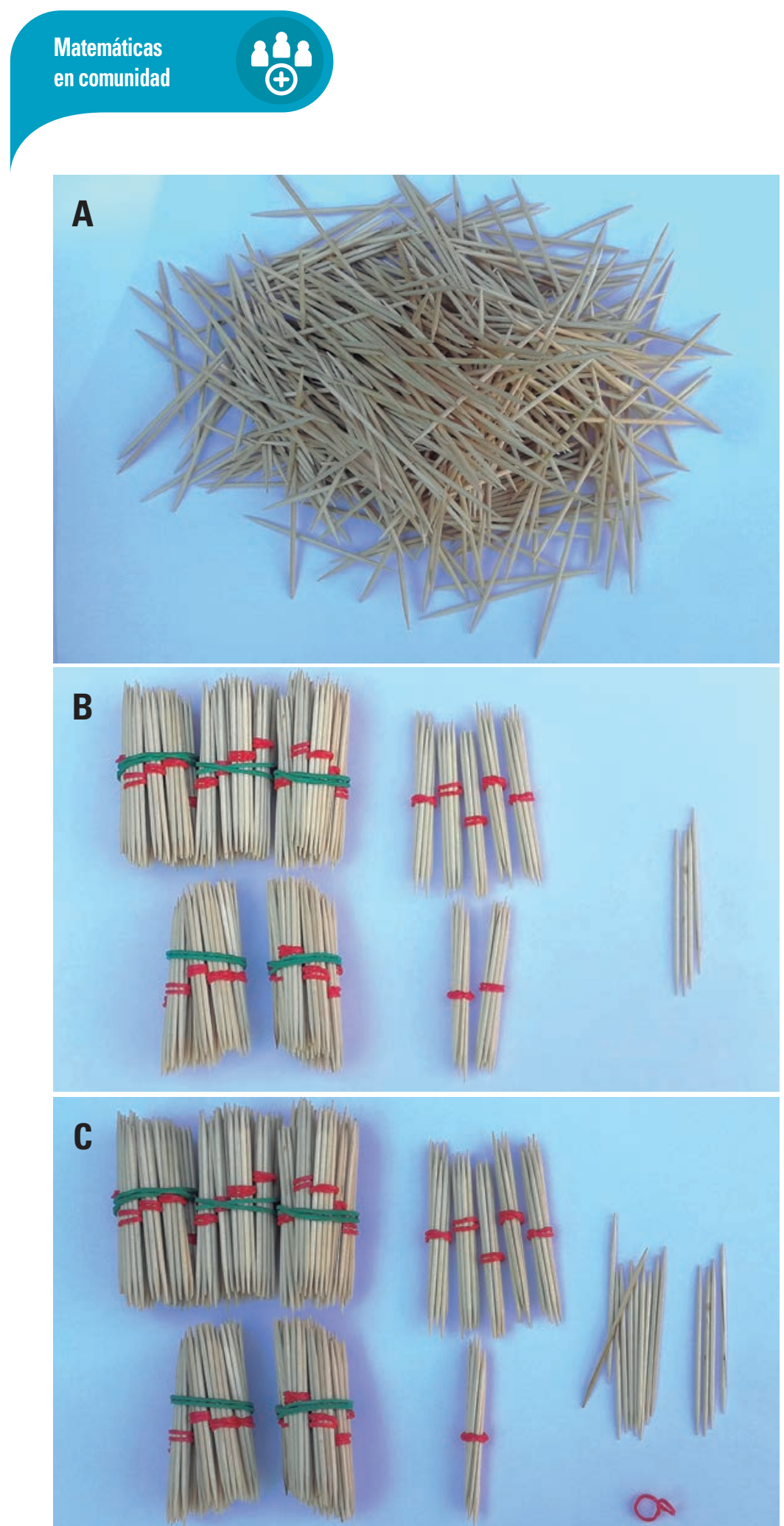

Estimamos, listos iya! A: ¿Cuántos crees que hay?

B: Agrupados de 10 en 10 vemos que hay 5 centenas, 7 decenas y 4 unidades

C: Preparamos el 574 para una resta en la que el sustraendo tuviera una cifra mayor que 4 en la posición de las unidades y lo reescribimos como quinientos sesenta y catorce tran distintos modelos y sistemas de representación. Se utilizan fotografías de contextos y objetos reales para ilustrar contenidos matemáticos y las figuras geométricas no están dispuestas siempre en la misma posición que suele coincidir con los lados paralelos a los bordes del papel.

* Los márgenes se utilizan relativamente poco y, cuando lo hacen, no es para promover aprendizajes memorísticos.
* Las ilustraciones son variadas y mues-
De hecho, los recuadros tipo "recuerda" deberían estar muy limitados y situados al final de la secuencia didáctica, después de que los estudiantes hayan comprendido lo que han descubierto, en caso contrario están indicando al alumno que utilice ese conocimiento y no pidiendo que relacione lo aprendido.

* En las páginas de desarrollo de contenidos, la maqueta no condiciona la secuencia didáctica y no se sigue siempre el mismo esquema: explicación teórica, ejemplos resueltos y actividades propuestas. La secuencia didáctica arranca con una propuesta de actividades guiadas que parte de casos concretos para permitir que los estudiantes "descubran" los contenidos matemáticos antes de ser nombrados con su terminología y nomenclatura abstracta.

* Los contenidos se presentan contextualizados con la vida diaria, relacionados con el resto de las disciplinas y enlazados con otros bloques de las matemáticas.

* Se facilita o se propone la utilización de materiales manipulativos y entornos virtuales que son llamados desde las páginas de contenido. No debemos conformarnos con que sean meramente un complemento o un taller en las páginas finales de cada unidad. Es necesario que desde el libro se invite a que el alumno "mida", "lance un dado", "coja el geoplano", "saque las regletas", etc.

* Las actividades de cada unidad o al pie de cada página, no son solo de aplicación de lo expuesto en esas páginas, sino que precisan de la utilización de otros aprendizajes previos. Por poner un ejemplo, si acaba de exponerse la multiplicación no todos los problemas que se realicen deberían resolverse multiplicando, sino utilizando otras estrategias vistas anteriormente en ese curso o en otros.

* Los problemas plantean contextos reales del alumno, cotidianos, ricos y significativos. Esto implica que no 
ponga al alumno en un contexto para que haga un procedimiento y automáticamente cambie en el problema siguiente a otro distinto.

* Aparecen retos de pensamiento, problemas abiertos, con varias soluciones e incluso sin solución. Se intuye que la finalidad de los problemas es provocar pensamiento y comunicación de las ideas, no meramente obtener "la solución" y que sea correcta. Esto requiere que haya ocasión para que los alumnos hagan conjeturas y estimaciones previas que provoquen la necesidad de ser comprobadas y contrastadas para que los errores puedan ser aprovechados como oportunidades para aprender a mejorar.

* Se incluyen autoevaluaciones y los resultados obtenidos ofrecen sugerencias para la realización de otras actividades de refuerzo o ampliación que suponen una oportunidad real para afianzar los conocimientos adquiridos o estimular nuevas ideas.

De todos modos, mientras llega el libro de texto de matemáticas "perfecto", ese ansiado tres estrellas Michelin, los profesores no podemos quedarnos de brazos cruzados. Hay muchas vías de actuación y todas pasan por conocer y valorar los puntos fuertes y débiles de cada propuesta editorial. De este modo estaremos en condiciones de luchar para tener en nuestros platos el menú que mejor se adapte a nuestros gustos y necesidades; también podemos tener una "biblioteca" de aula

\section{HEMOS HABLADO DE}

\section{Libros de texto; criterios para elección; matemáticas; indicadores didácticos; resolución de problemas.}

Este artículo fue solicitado por PADRES Y MAESTROS en mayo de 2018, revisado y aceptado en octubre de 2018.

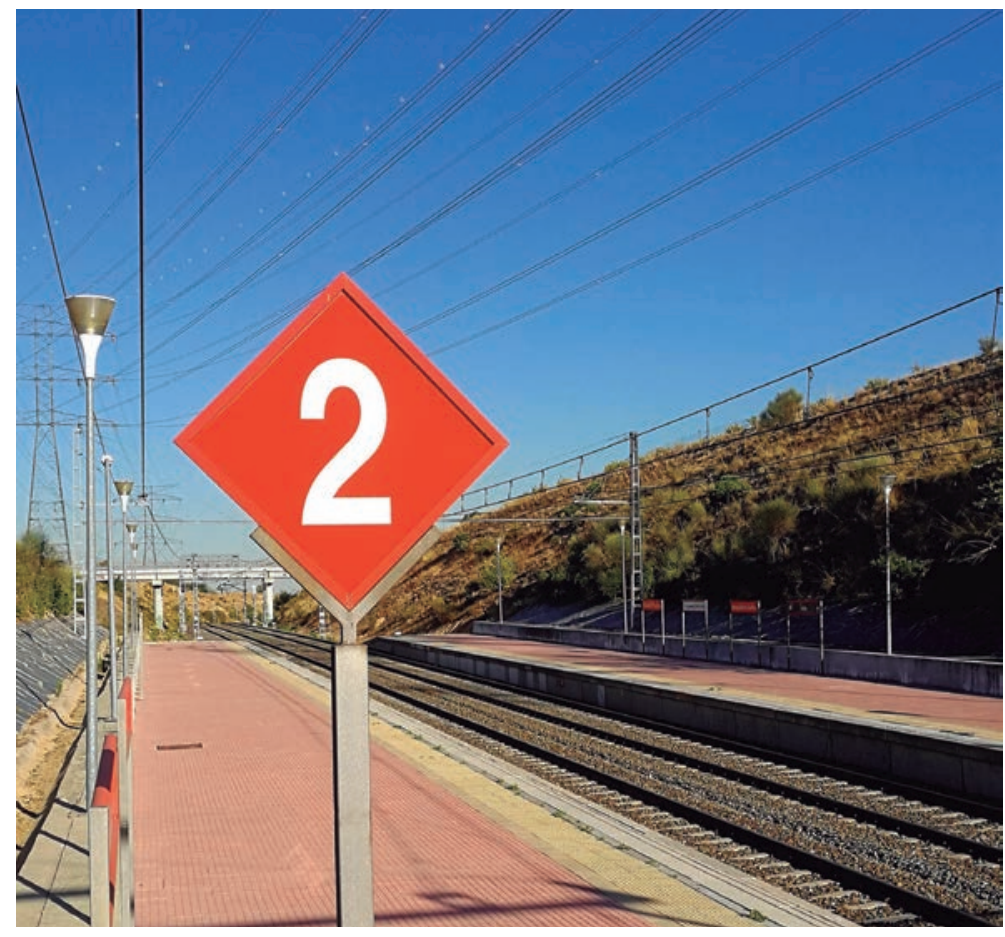

con una colección de varios ejemplares de cada editorial para hacer uso de ellos "a la carta" según los puntos fuertes de cada uno. Pero lo más importante es que, siendo conscientes de las carencias de los libros de texto y de las características que deberían tener, estaremos en condiciones de reclamar a las editoriales qué libros de texto de matemáticas queremos. Seguro que, si lo pedimos con insistencia y no nos conformamos con menos, lo acabamos consiguiendo. A fin de cuentas, podríamos pensar que las editoriales son cocineros que quieren conseguir que sus restaurantes (libros de textos) tenga tantas estrellas Michelin como sea posible, para tener bien satisfechos a sus clientes y atraer a muchos otros más •

\section{DD. DARA SABER MÂS}

Bourassa, M. (2013). Which One Doesn't Belong. Recuperado de http://wodb.ca

Cerezo, A., Calvo, C., y Barba, D. (2018). PuntMat. Recuperado de http://puntmat.blogspot.com/

MsldeasMnosCtas (2018). Más ideas, menos cuentas. Un blog sobre educación matemática. Recuperado de https://masideas-menoscuentas.com/ 